\title{
Comparative Study on Dimensional Stability of Polyvinylsilox- ane and Alginate as Interocclusal Recording Materials
}

\author{
Dr. Shahnaj Begum¹, Dr. Mahenaz Munira², Md. Fakrul Islam³, Hasina Mahmuda Ferdushi, \\ Dr. Alia Sultana ${ }^{5}$
}

Received: 01 October $2016 \quad$ Accepted: 12 November 2016

\begin{abstract}
:
Introduction: The fabrication of dental prosthesis requires the transfer of interocclusal records from patient's mouth to an articulator using different kinds of recording materials. Any inaccuracy in these interocclusal records leads to occlusal error in the final prosthesis. Interocclusal recording materials should have good dimensional stability for precise articulation. Purpose: The aim of this in vitro study was to evaluate and compare the dimensional stability of two types of interocclusal recording materials at various time intervals. Materials and Methods: The materials used in this study were polyvinylsiloxane (Reprosil Silicone Putty) and alginate (irreversible hydrocolloid). In this experimental study, specimen of polyvinylsiloxane and alginate were prepared from a custom made stainless steel die according to ADA specification no-19, in the form of a disk included three parallel lines on the surface which were provided as an indicator to see the dimensional stability of tested materials. The distance between parallel lines was measured at two fixed points using a traveling micrometer microscope. A total of 20 samples were made from group- $A$ and group- $B$ consisting of ten samples of each group. The measurements were made at time intervals of 1, 24, 48 and 72 hrs. Results: Two readings were taken for each sample at each time interval and the mean was considered to measure the dimensional change by comparing with that of the original measurements in the die. The results obtained were statistically analyzed using SPSS version 12 and paired $t$ test. The result of significance was expressed as $p$ value. $P$ value $<0.05$ was considered as significance. Both materials showed significant changes ie $<0.05$. Conclusion: Dimensional stability is influenced by both the "material used" and the "time" factors and was found to decreased dimensional stability as the time factors increased. Group A was dimensionally stable than Group-B.
\end{abstract}

Keywords:

\section{Introduction}

Inter occlusal records are the means whereby inter arch relationship are transferred from mouth to an articulator ${ }^{1}$. Maxillomandibular records are necessary to study the status of the dentition and to construct dental restoration. One type of record is used for mounting casts of the teeth or setting the articulator adjustments and another for appraising the degree of occlusal or incisal tooth contacts ${ }^{2}$. Adequate laboratory facilities are commonly not available locally and casts have to be sent to others laboratories for articu- lation. In these situations, the patients' interocclusal records are made and sent along with the cast to the laboratory. This requires that the records must be dimensionally stable for the given period of time before they are used to articulate the casts ${ }^{2}$. Recording maxillomandibular relationship is an important step in oral rehabilitation. This relationship is transferred to the articulator so laboratory procedure done on the cast will be corresponding with patients' mouth ${ }^{3}$. To create a harmonious occlusion, it is essential to record the existing maxillomandibular

1. BDS, FCPS (Prosthodontics) Assistant Professor, Dhaka Dental College Hospital, Dhaka.

2. BDS, MS (Prosthodontics), Assistant Professor, Shaheed Suhrawardy Medical College, Dhaka.

3. BDS, Consultant, Meem Dental Care, Mohakhali, Dhaka.

4. BDS, MPH, Assistant Professor, Dhaka Dental College Hospital, Dhaka.

5. BDS, DDS, MDS, Associate Professor, BSMMU, Dhaka.

Correspondence : Dr. Mahenaz Munira, email: munira78@gmail.com, cell: 01715388055 
relationship with the help of interocclusal recording materials. These materials should have good dimensional stability to achieve proper articulation ${ }^{4}$. Many materials are available for interocclusal record. These include: Bite registration wax (Aluwax, HiFi, baseplate), zinc-oxide eugenol paste, Addition silicone (polyvinylsiloxane), Polyether elastomer, Impression compound, Impression plaster of paris, Acrylic resin, Thermoplastic resin, Alginate (irreversible hydrocolloid), Condensation type silicone, Eugenol free zinc oxide eugenol paste ${ }^{4,5,6}$. Intercclusal recording materials are basically similar to impression materials but are modified to give good handing characteristics ${ }^{4}$. Each of these has advantages and disadvantages as interocclusal recording materials. In Bangladesh most popular interocclusal recording materials are alginate ${ }^{5}$. Alginate has limitation but it also has some advantages too over the other materials that make it more valuable. Because of its extreme fluidity before setting and its resilency after setting, alginate causes minimal tooth and tissue displacement when occlusal registrations are made with it ${ }^{5}$. Posselt thought that alginate records were superior to wax, but shrinkage made them useless after a few minutes ${ }^{7}$. The dimensional stability of interocclusal recording materials over time is of utmost importance, as it ensures a more accurate representation of the patient's maxillomandibular relationship ${ }^{10}$. So the study was done to compare the dimensional stability of polyvinylsiloxane and alginate at various times of intervals (1h, 24 hs, 48 hs, 72 hs).

\section{Materials \& Methods}

This comparative in vitro study was carried out Department of Prosthodontics, BSMMU, Dhaka, from June 2009 to 2010. Duplicated disk of polyvinylsiloxane and alginate obtained from custom made stainless steel die were used as a sample. The sample size of the study was twenty. Only freshly prepared duplicated disks from die were selected.

Group of sample:

Group A: 10 disks were made with polyvinylsiloxane. Group B: 10 disks were made with alginate.

Preparations of stainless steel die:

The stainless steel die had two portions: a round stainless steel test block and a stainless steel ring 4 that fits around the borders and acts as a mold for the specimen. A round stainless steel die was constructed for testing dimensional change. Three parallel lines were included on the die surface. These three lines were named $A, B$ and $C$ which were equally separated by a distance $3 \mathrm{~mm}$. The stainless steel ring that fits around the borders acts as a mold for the impression material. The thickness of ring was $0.3 \mathrm{~cm}$ and the diameter of the ring was $3 \mathrm{~cm}$. Therefore the stainless steel die include stainless steel ring and stainless steel test block. The distance between the two parallel reference lines $A$ and $C$ was measured at two fixed points. These reference points were scribed in the metallic die and were copied in the sample during their fabrication.

For polyvinylsiloxane specimen:

Two equal length of base and catalyst according to the manufacturers recommendation and kneaded with clean finger instead of wearing latex gloves to prevent sulfur contamination from these gloves which inhibits the setting of the addition silicone interocclusal recoding material and may produced major distortion. Then kneaded material together (approximately 45 seconds) until a uniform, streak free color was achieved. It was then placed on the surface of the die for impression making.

For alginate specimen:

For mixing alginate powder and water were measured according to the manufacturer's recommendation at room temperature. The measured powder $(9 \mathrm{gm})$ was shifted into premeasured water $(17 \mathrm{ml})$ that had already been poured into a clean rubber bowl. The powder was incorporated into the water by carefully mixing. Mixing time (30 seconds) was carefully maintained and after that it was placed on the surface of the die for impression making.

Sample collection:

After homogenous mixing, the materials were carried to the die. The stainless steel die was inverted on to a $4 \times 4$ inch square glass plate covered with polyethylene sheet. Hand pressure was applied for about five seconds initially to express the materials followed by application of a $500 \mathrm{~g}$ weight to further remove of excess materials. Each assembly remains for the manufacturer suggested setting time ie alginate for 2 minutes 20 seconds and additional three minutes to ensure polymerization of materials. The mold assembly was removed from the stainless steel die and all excess materials were trimmed. Samples were stored in room temperature. Later specimens were prepared in the form of a disk measuring $3 \mathrm{~cm}$ in diameter with three parallel lines on the surface.

Measurement of the test samples:

These three lines were named A, B \& C which were equally separated by a distance of $3 \mathrm{~mm}$. The distance between the two parallel reference lines, A \& $C$, were measured at two fixed points (A1C1 and A2C2). These reference points were scribed in the 
metallic die and were copied in the samples during their fabrication.

The distance between the two reference points of each sample (A1_C1, and A2_C2) were measured by a traveling micrometer microscope. It had a millimeter scale and a vernier scale which were attached together and with the help of vernier scale it was possible to measure up to 10 micrometer i.e. 0.01 millimeter. The two reference points between the vertical parallel line were measured through a magnifying tube attached with the traveling micrometer microscope. At first reference point A1 was placed beneath the magnifying tube on the platform of the microscope. The measurement M1 was recorded by the following formula. M1= (Reading of millimeter scale + Reading of vernier scale $x$ vernier constant i.e $0.01) \mathrm{mm}$. Then the platform was horizontally moved without shifting the sample and with the help of rotating the platform screw. Now reference point C1 was fixed under the magnifying tube. The measurement M2 was measured by the same formula. Measurement of the distance between A \& C parallel lines at reference point between $\mathrm{A} 1 \mathrm{C} 1$ is done by subtracting M1 from M2. So A1C1=M2-M1. In the same way, horizontal distance between A2_C2 was measured. The mean of two readings were used for calculation for each sample. Reading was recorded for all 10 samples of each group at intervals of $1 \mathrm{~h}, 24 \mathrm{~h}, 48 \mathrm{~h} \&$ 72 hours. The measurement data was collected from samples of each group and was recorded in data collection sheet. Horizontal linear distance between $\mathrm{A} 1 \mathrm{C} 1$ and $\mathrm{A} 2 \mathrm{C} 2$ is measured in millimeters.

Statistical analysis:

Data analysis was done by using computer based program SPSS (Statistical Package for Social Sciences) version 12. Paired t test was done to find out statistical significance value. The results were presented in tables and figures. The result of significance was expressed as $p$ value. $P$ value $<0.05$ was considered as significant.

\section{Results}

The present in vitro study was intended to compare the dimensional stability of polyvinylsiloxane and alginate at various times of intervals. Total 20 samples were evaluated. The findings of the study obtained were analyzed and presented below.
Table-l: Distribution of horizontal distance in group A

\begin{tabular}{|c|c|c|}
\hline Follow up & Number $(\mathbf{n})$ & Mean \pm SD $(\mathbf{m m})$ \\
\hline After one hour & 10 & $6.00 \pm 0.00$ \\
\hline After 24 hours & 10 & $5.97 \pm 0.38$ \\
\hline After 48 hours & 10 & $5.92 \pm 0.04$ \\
\hline After 72 hours & 10 & $5.82 \pm 0.04$ \\
\hline
\end{tabular}

GroupA: polyvinylsiloxane; n:Total number of sample Table-I shows that mean \pm SD was $6.00 \pm 0.00$ after one hour follow up visit $5.97 \pm 0.38$ after 24 hours, $5.92 \pm 0.04$ after 48 hours and $5.82 \pm 0.04$ after 72 hours follow up visit. It was indicated that maximum horizontal distance between A \& C after one hour follow up visit and minimum horizontal distance between $A$ \& $C$ after 72 hours follow up visits.

Table II: Distribution horizontal distance in different follow up of group $B$

\begin{tabular}{|c|c|c|}
\hline Follow up & Number $(\mathbf{n})$ & Mean \pm SD $(\mathbf{m m})$ \\
\hline After one hour & 10 & $5.68 \pm 0.05$ \\
\hline After 24 hours & 10 & $5.56 \pm 0.02$ \\
\hline After 48 hours & 10 & $5.49 \pm 0.03$ \\
\hline After 72 hours & 10 & $5.41 \pm 0.06$ \\
\hline
\end{tabular}

Group B : Alginate; $n$ :Total number of sample

Table-Il shows that mean $\pm S D$ was $5.68 \pm 0.05$ after one hour follow up visit, $5.56 \pm 0.02$ after 24 hours, $5.49 \pm 0.03$ after 48 hours and $5.41 \pm 0.06$ after 72 hours follow up visit. It was indicated that maximum horizontal distance after one hour follow up visits and minimum after 72 hours follow up visits.

Table III: Comparison of horizontal distance in group $A$ and group $B$

\begin{tabular}{|l|c|c|c|}
\hline \multirow{2}{*}{ Follow up } & Group A & Group B & \multirow{2}{*}{ P value } \\
\cline { 2 - 3 } & Mean \pm SD & Mean \pm SD & \\
\hline After 1 hour & $6.00 \pm 0.00$ & $5.68 \pm 0.05$ & $0.001^{*}$ \\
\hline After 24 hours & $5.97 \pm 0.08$ & $5.56 \pm 0.02$ & $0.001^{*}$ \\
\hline After 48 hours & $5.92 \pm 0.04$ & $5.49 \pm 0.03$ & $0.001^{*}$ \\
\hline After 72 hours & $5.82 \pm 0.04$ & $5.41 \pm 0.06$ & $0.001^{*}$ \\
\hline
\end{tabular}

Group A polyvinylsiloxane, Group B Alginate, The mean difference is considered significant if $p<0.05$. * Significant 
Table-III shows that after 1 hour follow up visit mean $\pm S D$ was $6.00 \pm 0.00$ in group $A$ and mean $\pm S D$ was $5.68 \pm 0.05$ in group $B$. After 24 hours follow up visit mean $\pm S D$ was $5.97 \pm 0.08$ in group $A$ and mean \pm SD was $5.56 \pm 0.02$ in group $B$. After 48 hours follow up visit mean $\pm S D$ was $5.92 \pm 0.04$ in group $A$ and mean $\pm S D$ was $5.49 \pm 0.03$ in group B. After 72 hours follow up visit mean $\pm S D$ was $5.82 \pm 0.04$ in group $A$ and mean $\pm S D$ was $5.41 \pm 0.06$ in group $B$. The difference was statistically significant $(P<0.05)$ between group $A$ and group $B$ in different follow up visits.

\section{Discussion}

The linear dimensional changes of two interocclusal recording materials were measured over time in this study. These measurements provided an indication of the dimensional stability of those materials. However, dimensional stability can also be studied in all the three planes using equipments like the condymeter, computerized Axitron and Buhnergraph ${ }^{4}$. Table 1 shows group A exhibited no significant difference between the die scribe and those of the sample at the immediate reading. Nisan et $\mathrm{al}^{2}$ observed that addition type silicone, polyvinyl siloxane is most accurate and stable interocclusal recording material. Table II shows in group B the same result. Table III shows comparison of horizontal distance between group $A$ and group $B$. The difference was statistically significant $(p<0.05)$ of all follow up visit between group $A$ and group B. Above reports showed similar results ${ }^{8}$. Some researchers carried out an experimental ${ }^{9}$ study and found that addition silicone presented smaller linear when compared to alginate. Moisture, especially, can cause considerable dimensional changes in alginate. Therefore great care is taken wrapping and packaging them during storage and transfer. Few authors have suggested ideal times for articulation of casts with respect to the type of interocclusal records used. The result of this present study was consistent with the above study. Thus, it becomes mandatory to choose a material depending not only on the clinical situation but also on the time taken for the articulation. From above study I found that dimensional changes of polyvinylsiloxane inter occlusal recording material was not significant in a horizontal plane after 1 and 24 hours. The changes after 48 and 72 hours were lesser than other group. So it can be concluded that polyvinylsiloxane is more dimensionally stable interocclusal recording materials than alginate.

\section{Conclusion}

In this study we concluded that dimensional stability is influenced by both Material and time factors. It decreased as the time factor is increased. Polyvinylsiloxane were dimensionally more stable than alginate interocclusal material.

\section{References}

1. Skurnik H. Resin registration for interocclusal recods. J ProsthetDent.1977;21(2):164-170.

2. Dua MP, Gupta SH, Ramachandran S, Sandhu HS. Evaluation for four elastomeric interocclusal recoding materials. MJAFII. 2007 ;63 (3) : 237-240.

3. Tripodakis AP, Vergos VK, Tsoutsos AG. Evaluation of the accuracy of interocclusal records in relation to two recording techniques. J Prosthet Dent.1997; 77 (2): 141-146.

4. Karithikeyan K, Annapumi. Comparative evaluation of dimensional stability of three types of interocclusal recording materials: an in vitro study. Journal of Indian Prosthodontic Society.2007; 7(1) :24-27.

5. Scott WR .Occlusal registration using alginate (irreversible hydrocolloid) impression material.J Prosthet Dent. 1978;40(5) :51 7-519.

6. Lassila V. Comparison of five interocclusal recording material. J Prosthet Dent.1986 ; 5: 215-218.

7. Eriksson A, Eriksson GO, Lockowandt P, et al .Materials for reliable interocclusal measurements. Br Dent J.2002 ;192 (7) : 385-400.

8. Lassila V, McCabe JF. Properties of interocclusal registration materials.J Prosthet Dent. 1985; 53: 100-104.

9. John J, Manapallil. Basic Dental Materials. 2nd ed .New Delhi: Jaypee Brothers medical publishers (p). Ltd.2003: 58.

10. Michalakis KX, Argiris $P$, Vassiliki A. Experimental study on Particular Physical Properties of several Interocclusal Recording Media. Part II: Linear Dimensional Change and Accompanying Weight Change. Journal of Prosthodontics. $2004 ; 13(3)$ : 150-159. 